\title{
Educare ad essere umani
}

\author{
Raffaele Beretta Piccoli, Scuola Universitaria Professionale della \\ Svizzera Italiana SUPSI
}

\begin{abstract}
L'articolo si avvia con un'analisi dell'esperienza dell'apprendimento focalizzata sui seguenti tratti essenziali: la relazione, la libertà, l'intelletto e la sfera affettiva. L'educazione è poi definita come accompagnamento del discente verso un'esperienza più profondamente umana della realtà. Questa definizione impone di giudicare la distinzione concettuale tra istruzione e educazione come riduttiva: l'istruzione è, infatti, essa stessa educante per il metodo, che insegna il confronto con i propri limiti e introduce a una posizione di apertura al reale, e per i contenuti, che arricchiscono di consapevolezza l'esperienza della realtà. In questo percorso è indispensabile l'educazione ai valori, tematizzata nella seconda parte dell'articolo con una proposta didattica di educazione alla cittadinanza democratica.
\end{abstract}

\section{Prima parte: apprendimento e educazione}

\section{A scuola: istruire o educare?}

Entriamo subito in un'aula scolastica per assistere a una scena: «Hai capito?» L'allievo sorride, scattante, lanciando un'occhiata ai compagni che stanno svolgendo un lavoro a coppie. Al suo fianco, una docente gli parla a voce bassa. «Sì risponde, ma l'insegnante intuisce che qualcosa ancora non va e rilancia: «Prova a raccontarmi tu, Riccardo, come ti viene... Parti da dove vuoi e cerchiamo di capire qualcosa di come le piante riescano a vivere». Silenzio. La docente tiene lo sguardo sul ragazzo, ride con gli occhi, accoglie e sostiene. "Guarda lo schema» con la punta della matita indica gli elementi del foglio su cui hanno appena lavorato. Silenzio. Anche le altre voci nella classe ora si smorzano e sul volto del giovane inizia a trasparire l'imbarazzo. "Non ti preoccupare - interviene l'insegnante - lo riguardiamo un'altra volta».

«E impossibile uscire da questo paradosso: - scrive Philippe Meirieu (2015) - imparare è fare qualche cosa che non si sa fare, facendolo» (p. 114). Imparare è lasciare le certezze per avviarsi al nuovo; imparare è correre sulla cresta che 
separa il "già» dal «non ancora»; è attravesarla lasciandosi alle spalle ciò che è famigliare. Su questa esperienza focalizziamo la prima parte di questo scritto: nelle prossime righe, cercheremo di coglierne alcuni aspetti nodali che ci serviranno per avvicinare una domanda classica della filosofia dell'educazione: la scuola dovrebbe limitarsi a informare, oppure dovrebbe assumere un onere educativo in senso più lato? La nostra analisi dell'esperienza dell'apprendimento, che svolgeremo aprendo ulteriori scorci sulla vita nella scuola, ci sarà di aiuto anche per chiarire il significato dei termini in gioco.

\section{L'apprendimento come adesione relazionale}

L'apprendimento è un'esperienza di natura intrinsecamente relazionale: il docente si relaziona con il discente, il discente si relaziona con il docente ed entrambi si relazionano con il mondo. Suscita stupore enumerare le attività che si svolgono in una scuola: gli allievi imparano a conoscere la natura che li circonda, il passato da cui scaturisce il loro mondo, il territorio in cui vivono; apprendono a ragionare e a calcolare; imparano ad esprimersi in lingue diverse, giocano insieme, compiono gite, disegnano, leggono, cantano, colorano, cucinano, scrivono, costruiscono, suonano, mangiano, cuciono e... Si potrebbe continuare. Ognuna di queste attività, a proprio modo, apre una finestra su uno spicchio di realtà: il mondo umano in tutti i suoi aspetti, come la creatività (arti), gli eventi e le idee del passato (storia), la corporeità (educazione fisica) e le convenzioni linguistiche; la logica razionale (matematica) e il mondo naturale (scienze). Gli alunni vivono tutto questo costruendo relazioni e amicizie. Qualche volta sfido i miei allievi: «Ditemi un altro luogo nel quale trovate tutte queste cose!» Un altro luogo del genere, semplicemente, non c'è. Un luogo che, potendo contare su risorse paragonabili, apra un giovane alla realtà del mondo. Ma questa apertura alla realtà implica quel passaggio verso l'ignoto citato in apertura il cui esito non è mai certo in partenza. Rientriamo in aula.

Settembre. Il vociare degli allievi si attenua quando appare, sulla soglia della porta, il docente di una nuova materia. Gli sguardi di molti allievi suggeriscono interrogativi: "Sarà simpatico?» "Sarà difficile?» "Riuscirò?» "Mi prenderanno in giro?». Altri sono più tranquilli, ma pure pronti a captare i primi indizi che trapeleranno dal nuovo arrivato. Inidizi come questo: "Guten Tag miteinander, ich bin der Deutschlehrer dieser Schule und ich wünsche Euch ein gutes Schuljahr». "Cos'ha detto?" chiede un'allieva alla compagna di banco che non risponde, perché focalizzata, a sua volta, su una domanda: «Ma io riuscirò un giorno a capire il tedesco e... A parlarlo?»

La sfida appare ardua e in essa sarà decisivo l'intervento della libertà: aderirà l'allieva alla proposta di lavoro del nuovo insegnante? Si metterà nella scomoda situazione di non capire niente, per iniziare a capire qualcosa? Questo passaggio non può essere evitato, l'allievo apprende attraverso la propria libertà, come ha ben illustrato Roberto Laffranchini (2015). Ciò non significa, naturalmente, che l'allievo abbia «un diritto al non lavoro», semmai egli ha il diritto all'educazione 
che implica il dovere dell'impegno, ma è semplicemente un dato di fatto antropologico: non si può obbligare qualcuno a imparare. Occorre che l'allieva si attivi, si implichi nel lavoro attraverso una libera mossa personale che non è scontata e che potrebbe anche non avvenire: perché? Per diverse ragioni. Ad esempio, l'allieva potrebbe manifestare opposizione verso gli adulti, magari proprio verso quell'insegnante che, chissà perché, la indispone. Oppure potrebbe essere fragile, così fragile da non reggere il confronto con il rischio implicato nella dinamica della libertà, perché escludere il tentativo, significa escludere la possibilità di scoprire che non si riesce, che, neppure questa volta, si è capita la lezione. Questa seconda ragione, l'ho colta con maggiore chiarezza grazie all'esperienza e al confronto con i colleghi: osservavamo alcuni allievi, come Riccardo, che non muovevano alcun passo malgrado il tempo che avevamo loro dedicato escogitando le più varie forme di semplificazione e di sintesi. «È debole, lo sappiamo - ci dicevamo - ma può essere così debole?» In un momento di chiarezza qualcuno, tra noi, ha intuito: «Sembra bloccato, preoccupato di nasconderci le proprie difficoltà, pensa che non le vediamo». In quell'occasione, c'era bisogno dell'adulto, dell'Insegnante e così, anche noi, abbiamo corso un rischio: «Non hai nulla di cui vergognarti». Lui ha annuito e da lì siamo partiti per costruire qualcosa su una base nuova o almeno... Per provarci.

\section{Sbagliare è giusto!}

Centrale, nell'esperienza dell'apprendimento, è la questione dell'errore. Si tratta di un aspetto delicato della relazione educativa al quale, giustamente, in ambito pedagogico si sta da tempo dedicando maggiore attenzione. Un maestro che ho incontrato nel mio percorso ripeteva spesso: «Sbagliate il più possibile!», perché ciò che conta è essere al lavoro. L'alternativa è presto detta: ritenere che sbagliare sia sbagliato e quindi avvertire l'errore come qualcosa che non dovrebbe esserci e che vada eliminato il prima possibile. Questa percezione implica fatalmente un giudizio: se è sbagliato sbagliare, io che sbaglio, sono sbagliato. E se è così, mi sento legittimato e stimolato a giudicare anche il mio compagno che commette un errore e con me lo faranno altri membri del gruppo, generando un circolo vizioso tra individuo e classe, tra giudizio interiore e giudizio esteriore, che ostacolerà la libera iniziativa del discente, il quale è invitato ad addentrarsi in terre sconosciute. È importante che il cambio di prospettiva avvenga già nella percezione dell'errore: ecco, c'è un'inesattezza, si è manifestata di fronte a tutta la classe, cosa si può fare? Anzitutto, la può constatare serenamente. Poi, sull'errore, il docente lavorerà con i propri allievi per compiere nuovi passi, prestando attenzione a regolare il giudizio per proteggere il benessere del gruppo.

Rieccoci in un'aula. Il docente sta per proporre alla classe una scenetta. Attorno a lui, gli allievi formano un cerchio sul pavimento dell'aula. Dopo aver introdotto l'attività, il docente si alza, prende la spugna dalla mensola della lavagna e la pone in mezzo al cerchio. "Guardate! - la indica - cosa fa una spugna?» «Pulisce?» Propone timidamente un allievo, «assorbe?» Precisa una ragazzina. 
«Esatto! - conferma l'insegnante - la spugna pulisce e assorbe. Ma questa continua - è una spugna speciale che assorbe i giudizi». Gli allievi restano in silenzio e rimettono a fuoco l'oggetto posto di fronte a loro. "Fare una scenetta, come abbiamo visto, può fare paura a qualcuno di noi: facciamo assorbire alla spugna questi nostri timori!» Così, un allievo alla volta, in ordine sparso e senza alcun obbligo, iniziano a dichiarare ad alta voce verso la spugna i propri timori: «Ho paura di sbagliare!» "Ho paura di non sapere cosa fare!» "Ho paura che i compagni ridano!». Al termine dell'attività, l'insegnante prende la spugna e tenendola in mano conferma: «È bella pesante ora, ha assorbito tutto», apre la finestra, la appoggia sul davanzale e richiude le ante. «Non ci sono più giudizi qui - conclude - gli errori, i vuoti, i gesti incomprensibili sono tutti i benvenuti e ci permettiamo di lasciarli in pace, lasciamoci in pace».

\section{Apprendimento, affettività e discipline}

Già si è intuito quanto, nell'apprendimento, sia rilevante il ruolo delle emozioni. La storia della filosofia non sempre ci aiuta a cogliere questa importanza: Socrate, Platone, ma anche Cartesio con i suoi colleghi razionalisti ci hanno abituati a una sorta di «monopolio gnoseologico della ragione». Secondo questi autori, infatti, la conoscenza avrebbe a che fare solo con la «testa». Di segno opposto, ma con le medesime conseguenze, è l'empirismo adottato da pensatori come David Hume, che riducono la conoscenza a una questione di «sensi» e di «percezione». Non si esce da quell'opposizione che Lina Bertola (2015) ha giustamente chiamato «ontologia della separatezza» (p. 63). Le nostre visite nelle aule ci hanno già mostrato che le cose non stanno così:

C'è una priorità logica e psicologica della dimensione emotiva e motivazionale su quella cognitiva: la nostra mente apprende bene solo tutto quello che appare importante, interessante, stimolante, utile, necessario. (Polito, 2015, p. 22)

Per apprendere bene occorre anche un coinvolgimento emotivo. Mario Polito (2015) ci ricorda che le emozioni non possano essere prodotte, per così dire, artificialmente, ma devono scorrere genuinamente dal docente verso l'allievo. L'insegnante emoziona se lui stesso è emozionato, stupisce se prova stupore per ciò che insegna. Questa considerazione è carica di conseguenze: è importante, certo, curare la formazione pedagogica dell'insegnante ma questo lavoro deve potersi fondare sulla passione e sull'approfondimento della disciplina, perché le motivazioni più forti per l'apprendimento sono di natura intrinseca, ovvero, sono legate a ciò che si sta imparando, al valore che assume agli occhi di chi impara (Nucci, 2002). Come ci ricorda Rousseau $\left(1762^{2}\right.$, 2017), questo valore può essere "sia di diletto, sia di utilità» (p. 121): si può essere motivati ad imparare perché serve a qualcosa oppure, e non bisogna sottovalutare questo secondo aspetto, perché è bello, perché affascina ed è interessante. S'intuisce allora facilmente l'autogol rappresentato dalle "contrattazioni scolastiche» (Meirieu, 2013, p. 40): contrattare l'attenzione degli allievi in cambio di una successiva attività 
meno impegnativa significa, come ha ben illustrato Meirieu, confermare l'ipotesi degli allievi secondo la quale il lavoro che si sta affrontando non abbia in sé un valore sufficiente per motivare la fatica e quindi che sia necessario ricorrere a motivazioni estrinseche. E nel lavoro rientra sì il contenuto dell'apprendimento, sia esso un sapere o un'abilità, ma anche l'esperienza dell'apprendimento che implica tutti i fattori illustrati fino a questo momento, a partire dal serrato confronto con i propri limiti.

Abbiamo usato una parola che non è certo di moda ai nostri tempi: «la fatica». Anch'essa rappresenta un ostacolo importante alla mossa della libertà dell'allievo, alla sua adesione al lavoro. Accompagnare ad attraversare la fatica limitando le scorciatoie delle motivazioni estrinseche rappresenta una sfida centrale della professione dell'insegnante: constatare la fatica, condividerla e appoggiarla sulle risorse del gruppo, incoraggiare, mostrare la dignità del lavoro e la promessa di soddisfazione che esso porta con sé.

\section{La Libertà dell'insegnante}

$\mathrm{Ci}$ siamo avvicinati in queste righe al nucleo della relazione educativa che vede un delicato incontro tra due libertà. Sì, perché, se all'apprendimento è indispensabile la libera mossa del discente, lo è altrettanto quella dell'insegnante. L'insegnante che si mette in gioco, che si appassiona per ciò che insegna e che cerca le modalità più consone e più creative per stimolare i propri allievi. Ciò significa che la libertà d'insegnamento e l'autonomia didattica ${ }^{2}$ non rappresentano una semplice concessione all'arbitrio dell'insegnante, bensì che siano condizioni perché egli possa assumersi pienamente le responsabilità che gli competono, portando se stesso nella relazione educativa e adattando il lavoro alle circostanze che incontra. Questa responsabilizzazione, come ha giustamente sottolineato Alessandro Mariani (2011), implica «un celere abbandono della visione corrente delle professionalità formative, viste come esecutrici di modelli pedagogici standardizzati e programmi preconfezionati» (p. 79): la professione dell'insegnante non ha una natura meramente tecnica; non si tratta, per il docente, di fare determinate cose secondo indicazioni formulate a priori, bensì di coinvolgere la propria umanità, senz'altro rispettando una deontologia e un'etica professionali, in una relazione educativa che richiede autenticità, integralità, margini di scelta e di adattamento.

La libertà d'insegnamento è necessaria all'insegnante che deve potersi mantenere scomodo nella tensione tra l'educabilità e la libertà dell'alunno (Meirieu, 2015). Come nell'esempio visto dell'allievo che abbiamo chiamato Riccardo: sa quando compiere un passo in avanti e insistere ancora un po' e sa quando ritirarsi e rimandare perché è troppo. Il docente è umile di fronte al mistero rappresentato dalla persona libera perché, come ci ricorda Rousseau $\left(1762^{1}, 2017\right)$, «si conosce $[\ldots]$, o si può conoscere, il punto iniziale da cui parte ciascuno di noi per arrivare al livello comune di intelligenza; ma chi mai conosce l'altra estremità?» (p. 74). È di attualità questo avvertimento dell'autore 
illuminista: "Non so che alcun filosofo abbia avuto fino ad oggi il coraggio di dire: ecco il termine cui l'uomo può arrivare e che non saprebbe sorpassare» (p. 74). La posizione del docente è, come si diceva, scomoda, perché ha a che fare con l'instancabilità: egli è chiamato fino all'ultimo a credere nelle possibilità del proprio allievo, ma lo deve fare nel rispetto della sua libertà. Quanti dilemmi scaturiscono nella quotidianità da questa tensione: quante scelte da prendere, quante riflessioni, quanta autocritica, quanta pazienza.

Ecco una nuova scena di vita scolastica. Ma non ci troviamo in un'aula, bensì nello studio di un'insegnante. È la sera di una domenica soleggiata trascorsa a correggere un compito in classe. Posando la penna, la docente libera un sospiro: quattordici allievi su ventuno non hanno raggiunto la sufficienza. Non è la prima volta che capita con la classe ed è innegabile: la docente si deve confrontare con la frustrazione. Che fare? «Riproviamocil!» Si dice, con la mente che già sta escogitando una nuova proposta: a ogni lezione distribuirà delle carte da gioco che, in forma interrogativa, riassumono $\mathrm{i}$ concetti chiave appena trattati. Ai ragazzi è chiesto di completarle al termine degli incontri, oppure a casa e di lezione in lezione, l'insegnante le ritirerà, le correggerà e discuterà i problemi generali con la classe, quelli particolari con gli interessati. Si parte. Alcuni allievi aderiscono immediatamente, altri, molti altri, no: perdono le carte, non le completano e giustificano con insistenza le loro inadempienze. La docente parla con ognuno di loro, li trattiene in pausa e cerca di spiegare che non può sostituirsi al loro lavoro. Quando le mancate consegne si reiterano contatta i genitori, spiega il lavoro che sta proponendo e chiede la loro collaborazione. La verifica successiva? Insufficienze dimezzate. L'insegnante è di nuovo nel suo studio. Piuttosto che fornire risposte definitive, questa esperienza ha suscitato in lei ulteriori domande: fino a che punto è opportuno e educativo insistere? Di chi è la responsabilità delle insufficienze degli allievi se il docente non riesce - come non può riuscire - ad adottare una pratica di questo tipo per tutte le classi? E se l'adotta solo con la classe che riscontra maggiori difficoltà, non genera un'ingiustizia rispetto alle classi parallele?

Dilemmi. Aggiungiamo una considerazione indispensabile. L'insegnante, lo abbiamo detto, non sa dove potrà arrivare un allievo nel suo percorso di vita, tuttavia è possibile che gli tocchi constatare che con lui, nel tratto di strada in cui gli è stato accanto, quel passo non è stato in grado di compierlo: «può succedere e, di fatto, succede» (Laffranchini, 2015, p. 36). Si vede che questo libro è nato dal terreno: una relazione educativa che pretenda la certezza del successo da qualche parte cade nel riduzionismo, ovvero, elude la complessità, ma anche l'integralità umana implicata nell'apprendimento. Il docente non può, per definizione, garantire il conseguimento degli obiettivi di tutti i suoi allievi; l'unico modo per farlo sarebbe rinunciare alla proposta di un lavoro, di un passo autentico e ciò, oltre a falsare la natura stessa dell'apprendimento, implicherebbe fatalmente l'invio all'allievo di un messaggio di scarsissima stima: «Tu non puoi farcela, né ora né in futuro, quindi, risolvo io, al tuo posto, il problema di 
matematica su cui sei chinato; oppure ti dò io una valutazione positiva anche se, lo sappiamo bene entrambi, non l'hai raggiunta con il tuo lavoro».

\section{L'educazione in senso Lato}

Siamo a Zurigo. È un fine settimana e Riccardo, che ha ormai raggiunto la terza media, sta visitando la città per la prima volta, con la propria famiglia. A metà di una gradinata si ferma improvvisamente e alza lo sguardo sulle due torri del Grossmünster. Gli occhi si dilatano: «Che bello!» «Cos’è?» Chiede la sorellina. «E una chiesa» suggerisce il papà. "Sì - mette a fuoco Riccardo, portandosi l'indice alle labbra - una chiesa cristiana... Sapete? - aggiunge - il cristianesimo si è diffuso in Europa alla fine dell'Impero romano, verso il 300 o il 400 se non sbaglio. All'inizio i Romani non lo hanno accettato e molti cristiani sono stati uccisi, poi sì e anzi, è diventato obbligatorio e per molti secoli non si è potuta scegliere la propria religione». "È vero» conferma il padre di Riccardo, lasciando trapelare un certo stupore. "Guarda! - Riccardo indica un cartello bianco e azzurro - questa è «Piazza Zwingli! Certo, qui è iniziata la Riforma protestante di Zurigo che poi si è diffusa in molti altri cantoni, quindi è una chiesa protestante!» Lo sorellina lo guarda. "Anche tra protestanti e cattolici purtroppo aggiunge Riccardo - c'è stata tanta violenza». Dopo qualche istante di silenzio, abbassando lo sguardo dal duomo ai suoi famigliari, Riccardo conclude: «È bella la libertà che abbiamo oggi».

A questo punto della nostra riflessione dobbiamo chiederci in termini più espliciti cosa significhi «educare». $\mathrm{Ci}$ viene in aiuto un filosofo, che fu anche scienziato, teologo e imprenditore; un vero e proprio genio poliedrico del Grand Siècle francese: Blaise Pascal. Prendiamo spunto da uno dei passaggi più celebri delle sue annotazioni sparse, trovate cucite a mazzetti dopo la sua morte:

L'uomo non è che una canna, la più fragile di tutta la natura; ma è una canna pensante. Non occorre che l'universo intero si armi per annientarlo: un vapore, una goccia d'acqua è sufficiente per ucciderlo. Ma quand'anche l'universo lo schiacciasse, l'uomo sarebbe pur sempre più nobile di ciò che lo uccide, dal momento che egli sa di morire e il vantaggio che l'universo ha su di lui; l'universo non sa nulla. (Pascal, 1670¹, frammento 231 (classificazione di Philippe Sellier) o 347 (classificazione di Léon Brunschvicg)

L'essere umano, ci dice Pascal, nella propria fragilità e piccolezza, ha qualcosa di grande: è consapevole di sé e della realtà che lo circonda ${ }^{3}$. Pascal parla di questa potenzialità come di una differenza specifica, essenziale, che distingue gli uomini dalle altre entità che li circondano. Una differenza che lo distingue e gli conferisce una dignità che non si misura in termini di forza o di grandezza, bensì, in quella che potremmo definire profondità dell'esperienza. Ecco, l'educazione è quel percorso, caratterizzato dai fattori che abbiamo descritto, che conferisce profondità, in termini di solida consapevolezza, all'esperienza e che, facendo ciò, la arricchisce e la rende più umana. Rende l'essere umano, più umano (Bellamy, 2016). L'educazione introduce (Giussani, 2005; Jungmann, 
1939), apre la persona a un'esperienza della realtà, compresa la propria persona, più consapevole, più profonda, più ampia, più chiara, attraverso l'intervento di un'autorità, ovvero di una persona che, di questa esperienza, è più esperta; nell'ottica comunque di una progressiva, ma mai completa, autonomizzazione. Perché questo avvenga compiutamente, è indispensabile un'acquisizione di valori trasmessi dall'educatore: non bastano conoscenze e abilità, ma è necessario il confronto con l'etica. Lo abbiamo visto nell'esempio di Riccardo: nelle sue affermazioni colme di stupore le conoscenze storiche erano unite, quasi fuse, a giudizi di valore: «È bella la libertà che abbiamo oggi!».

Secondo l'articolo 2 della legge della scuola del Canton Ticino, l'istituzione scolastica ha principalmente una doppia finalità: trasmettere cultura e educare a realizzare valori. Sono menzionati, in particolare: la giustizia, la libertà, la pace, la parità tra uomo e donna, l'integrazione sociale, il rispetto dell'ambiente e gli ideali democratici. Principi che hanno quale fondamento l'etica universale, radicata nella persona stessa, che ispira la definizione dei «diritti umani» descritti ad esempio nella Dichiarazione promossa dall'ONU nel 1948, dopo gli orrori della Seconda guerra mondiale, e nella successiva Convenzione Europea per la salvaguardia dei diritti dell'uomo e delle libertà fondamentali. Non c'è spazio per il relativismo: si tratta di valori e diritti che rientrano a pieno titolo nei principi fondativi dell'istituzione scolastica. Quando la scuola, come accade nei regimi totalitari, si smarca da questa vocazione educativa, tradisce i propri principi (Meirieu, 2015). Non bisogna trascurare neanche i valori estetici; il ragazzino a Zurigo di fronte al duomo esclama "che bello!»: l'esperienza della bellezza in senso lato, bellezza della natura, bellezza dell'arte e di tutte le discipline, ma anche bellezza del lavoro, della condivisione e della tolleranza, è educativa; educa la persona a entrare in un rapporto più profondo e significativo con se stessa $\mathrm{e}$ con tutta la realtà.

Questa è la portata dell'educazione, non meno. Occorre tenerlo presente quando ci si confronta con elenchi di "competenze per la vita», come quello proposto dall'Organizzazione Mondiale della Sanità ${ }^{2}$ : documenti che senz'altro mettono a fuoco alcuni cardini dell'esperienza educativa, quali il "pensiero critico» o "l'empatia», ma che potrebbero indurre a una traduzione troppo tecnica di una sfida che ha l'ambizione di contribuire a realizzare l'umanità delle persone attraverso la cultura e la realizzazione, viva, di valori.

\section{Nella scuola, istruire o educare?}

Torniamo allora alla domanda d'apertura: la scuola deve istruire o educare? Se per «istruire» intendiamo «informare», secondo i criteri proposti dalla legge della scuola del Canton Ticino, trasmettere la cultura in termini critici, scientifici e pluralisti, data la visione dell'educazione che abbiamo proposto, ci rendiamo conto immediatamente che questa domanda semplifica eccessivamente i termini del problema. Considerando il legame che unisce l'educazione ai valori, potremmo chiederci: la scuola deve solo trasmettere informazioni e abilità $o$ 
anche valori? La risposta è assodata: la scuola educa ai valori della democrazia. Tuttavia, approfondendo la riflessione, ma anche più semplicemente entrando nelle aule di scuola e assistendo al lavoro che vi si svolge, appare evidente che la distinzione tra «istruzione» e «educazione» ha limiti importanti: l'educazione, intesa come cammino di approfondimento dell'esperienza della realtà tramite l'attualizzazione delle potenzialità umane, integra e implica l'istruzione come metodo e come contenuti.

Partiamo dal metodo. Abbiamo analizzato alcune importanti implicazioni prettamente antropologiche dell'esperienza dell'apprendimento: l'incontro tra le libertà dell'insegnante e del discente, il confronto con il rischio, con i limiti e la fatica, l'implicazione della dimensione affettiva accanto alle facoltà intellettuali. Apprendere ad apprendere, implica il confronto con tutti questi aspetti per la ricerca di un equilibrio sempre più aperto al nuovo e questa è un'esperienza profondamente educativa che vede al proprio cuore la relazione: quella tra l'insegnante e l'allievo; quella tra l'allievo e l'allievo e quella tra insegnante e allievo da una parte e il resto della realtà dell'altra. Da questo punto di vista tutte le discipline sono educative.

Questo lavoro mette in relazione l'allievo con il mondo, cioè, con dei contenuti disciplinari che, senza voler scandalizzare nessuno, è opportuno anche studiare a memoria:

Non c'è niente di più bello dell'imparare a memoria [...] - afferma FrançoisXavier Bellamy - Imparare a memoria significa lasciare che un testo, una musica, un sapere vengano dentro di noi, ci trasformino, ci elevino e amplino la nostra mente e il nostro cuore fino alla loro altezza. (Bellamy, 2016, pp. 121-122)

Il filosofo e insegnante francese coglie un aspetto importante che tutti noi, pensando alla nostra esperienza di studio, possiamo confermare: studiare a memoria un contenuto non significa appiccicare provvisoriamente un corpo estraneo alla propria persona, come accade forse più facilmente con l'immediatezza delle informazioni che si ricavano in internet, bensì, iniziare un dialogo interiore che produce associazioni e collegamenti e che cresce assieme all'esperienza, come nell'esempio del duomo di Zurigo. Scoprire di saper cogliere il significato di una frase in lingua straniera, magari rubata a un passante; scoprire di saper contestualizzare l'esperienza religiosa di una donna velata incontrata alla cassa di un supermercato, riconoscere con meraviglia i processi naturali che caratterizzano un cambio di stagione, aver sperimentato uno sport che si vede in televisione durante le Olimpiadi, saper giudicare la frase dal retrogusto intollerante verso una cultura diversa che riporta un giornale... Così i contenuti delle discipline educano a un'esperienza più umana della realtà. Così, imparando un metodo per camminare e dei contenuti lungo il cammino, l'allievo vive una crescita integrale che coinvolge tutte le sue facoltà. Così, più che con un corso specifico $^{5}$, egli impara anche a prendere decisioni, confrontandosi con i valori, con le conoscenze, con le emozioni e con l'intera propria umanità. 
La scuola dunque educa e istruisce, o meglio, educa istruendo e istruisce educando, unendo indissolubilmente queste due dimensioni in un lavoro di natura primariamente relazionale che tocca i più vari strati antropologici della persona.

\section{Seconda parte: educazione alla cittadinanza democratica}

\section{Cittadinanza democratica: un'educazione antropologica} Nella seconda parte di questo articolo ci occupiamo di un' «educazione» di grande attualità nella scuola ${ }^{6}$ : quella alla cittadinanza democratica, provando ad applicare alcuni dei risultati che sono scaturiti dalle riflessioni precedenti. Questa educazione mira principalmente alla trasmissione dei valori e quindi alla sensibilizzazione ai diritti fondamentali che fondano la convivenza democratica ${ }^{7}$ che abbiamo precedentemente citato: la pace, la giustizia (o il bene), la libertà, la tolleranza, il rispetto dell'altro e dell'ambiente. Che sfida impegnativa e che responsabilità importante per un docente! Come si fa a promuovere un'educazione che ha degli obiettivi di questa portata?

Un'educazione alla cittadinanza, in primo luogo, a nostro avviso, deve mantenersi prossima alla dimensione antropologica in tutte le sue sfaccettature: quando si parla di valori e di diritti, infatti, si ha a che fare con i livelli più profondi dell'umano. Eे qui, del resto, che si pone il concetto stesso di «democrazia»: «Una democrazia è qualcosa di più di una forma di governo - osserva John Dewey (1954) - è prima di tutto un tipo di vita associata, di esperienza comunicata e congiunta» (p. 116).

Ogni generazione la [la democrazia] deve realizzare sempre di nuovo per se stessa; [perché] la sua vera natura, la sua essenza, è qualcosa che non può essere passata da una persona o da una generazione all'altra, ma che deve essere elaborata in termini di bisogni, problemi e condizioni della vita sociale della quale siamo parte. (Dewey, 1938, p. 300)

La democrazia non si eredita (Silva, 2015), ma deve essere ricostruita da ogni generazione. Ciò è possibile, solo se i valori che la ispirano sono assimilati profondamente dal giovane, radicati in lui perché compresi nella loro reale portata e quindi liberamente accolti fino a determinare l'agire. Sembra alludere proprio a questo un passaggio importante di una raccomandazione formulata dal Consiglio d'Europa:

Uno degli obiettivi fondamentali di tutta l'educazione per la cittadinanza democratica e per l'educazione ai diritti umani non è solo dotare gli studenti di conoscenza, comprensione e competenze, ma anche conferire loro la prontezza ad agire nella società, nella difesa e nella promozione dei diritti umani, della democrazia e dello Stato di diritto. (Raccomandazione 7 del Comitato dei Ministri degli Stati membri del Consiglio d'Europa sull'educazione alla cittadinanza democratica, 2010) 
Siamo di nuovo a scuola. Con determinazione e fatica un docente è riuscito a costruire nel tempo un clima di lavoro ordinato: gli allievi alzano la mano per intervenire, restano in silenzio quando qualcuno ha la parola e chiedono il permesso per spostarsi dal banco. Ed ecco, un allievo, uno di quelli che rispetta abitualmente tali disposizioni, s'avvicina all'insegnante e ottiene il permesso di andare in bagno. E là riversa tranquillamente una parte dei propri bisogni sul pavimento.

Dovrebbe far riflettere lo stato in cui si trovano regolarmente i bagni di alcune scuole perché, se la cittadinanza si riduce a regole di buona educazione che si dimenticano già al primo metro percorso oltre la porta dell'aula, là dove l'allievo non si rende conto che qualcuno dovrà riparare il suo danno, significa che l'educazione scolastica resta inincidente e umanamente astratta, fondata su motivazioni estrinseche come l'evitamento dei rimproveri: caduto il rischio di essere colti in fallo, cadono anche le ragioni per rispettare le regole. Ulteriore indice di questa astrazione è la scissione tra il dire e il fare che si riscontra facilmente nelle discussioni in classe: provate a chiedere ai ragazzi quali siano le regole da rispettare in aula; anche i membri della classe più indisciplinata sapranno elencarle con precisione, naturalmente, senza rinunciare, neanche in quel preciso momento, al consueto clima di disattenzione e mancanza di rispetto per l'altro.

A questo proposito, ci sembra particolarmente utile un'esperienza che Edgar Morin ha lucidamente definito come "comprensione umana»:

[La comprensione umana] riconosce l'altro nello stesso tempo come simile a sé e differente da sé: simile a sé per la sua umanità, differente da sé per la sua singolarità personale o/e culturale. Il riconoscimento della qualità umana dell'altro è una precondizione indispensabile a ogni comprensione. (Morin, 2015, p. 51)

La tradizione tomistica la chiamava "conoscenza per connaturalità»: io riconosco tra me e te una comune natura e attraverso di essa comprendo intimamente la tua esperienza. «Se vedo un bambino in lacrime - esemplifica Morin - lo comprenderò non misurando il grado di salinità delle sue lacrime, ma ritrovando i miei sconforti infantili» (p. 50). Così si produce una vicinanza antropologica che può fungere da base per costruire una reale comprensione dell'altro: della sua esperienza e del suo vissuto.

In questo percorso esplorativo dell'umano s'inserisce l'educazione ai valori. $\mathrm{Si}$ tratta di un cammino lento e progressivo che, passo per passo, tenta di portare gli allievi a un'intuizione sempre più limpida: i valori e i diritti democratici rispondono a esigenze radicate nella propria umanità e in quella dell'altro. All'intuizione sperimentata che realizzare questi valori, tendenzialmente desiderati da ognuno, rende più bella - ecco il legame con i valori estetici - più intensa e più umana l'esperienza, propria e degli altri.

Educare alla cittadinanza, in quest'ottica, significa principalmente creare ponti di comprensione umana tra le persone e orientarli attraverso l'educazione ai valori, sulla cui base, sarà finalmente possibile riconoscere, accettare e apprezzare tutte le differenze che non entrino in contrasto con i diritti fondamentali e con i principi della democrazia: le differenze dell'altro, ma anche, grazie alla duttilità introdotta dalla comprensione, quelle che io ho per rapporto a lui. 
S'intuisce facilmente quanto, in tale ottica, diventi centrale la relazione umana tra l'insegnante e il discente. Come ha osservato Alessandro Mariani, l'educazione alla cittadinanza democratica richiede «una maggiore responsabilizzazione (che significa anche più potere e più autonomia) dei maestri, dei professori, degli educatori e dei pedagogisti affinché possano assumere - de jure e de facto - il ruolo di intellettuali pubblici» (Mariani, 2011, p. 79). Occorre, più che mai, quella libertà che permetta al docente di svincolarsi dalle riduzioni tecnicistiche dell'insegnamento, per portare se stesso nella relazione educativa, testimoniando i valori e la comprensione ai quali ha il compito di educare.

Proponiamo ora due strade che, in modi diversi, possono contribuire e realizzare nella scuola un'educazione ai valori democratici ancorata alla dimensione antropologica: l'incontro con figure significative della storia umana e la valorizzazione della quotidianità.

L'incontro con figure significative della storia umana L'affezione ai valori universali

La Guerra del Sonderbund è al centro di una lezione: un passo il docente e un passo la classe, ci si introduce all'evento, leggendo qualche riga di testo, guardando qualche istante di documentario, riprendendo i testi, analizzando documenti e cartine. È un'operazione delicata, quasi chirurgica: può funzionare oppure no, strada facendo l'esito può variare, l'attenzione può essere schiacciata dalla stanchezza o dalla fatica e alla concentrazione può sostituirsi il disimpegno. Il docente lo sa: se succede, resta poco da fare: lui è da solo, loro in ventiquattro. A tratti, l'insegnante avverte dei cedimenti e s'impegna con energia per sostenere il lavoro: riprende il discorso, lo riassume brevemente, cerca di riattizzare la curiosità... «Si arriverà o no alla guerra?» "Chi vincerà?» «E noi, il Ticino, da che parte saremo?». S'incontra la figura di Guillaume-Henri Dufour e il dramma della scelta: la guerra contro i connazionali o il tradimento dei doveri verso la patria? D'un tratto e inaspettatamente, l'insegnante, raccontandolo, si rende conto di essere coinvolto da questo personaggio, toccato dalla sua verità e in modo ancora più inaspettato, ma chiarissimo, si accorge che anche la classe di quattordicenni lo è. Lo sente e lo vede: c'è una grande attenzione. Ci sono occhi lucidi.

Ciò che è avvenuto in questa lezione è un vero e proprio incontro umano. Tra il docente e gli allievi, ma soprattutto tra di loro e la vicenda drammatica di un personaggio che spicca, nella storia svizzera, per la propria adesione ai valori fondamentali: un generale che avverte la drammaticità umana implicata in una guerra civile, che cerca di ridurre le vittime e che partecipa alla creazione del Comitato Internazionale della Croce Rossa. Come ogni incontro autentico, muove più strati della persona: quello intellettuale, ma anche quello affettivo. Un incontro di questa intensità, come abbiamo precedentemente osservato, può essere sì preparato dal docente, tuttavia, non può essere imposto: in un qualche modo accade e certamente non rappresenta la quotidianità. È qualcosa cui tendere, apparecchiando regolarmente occasioni propizie. 
L'apprendimento che accade come esperienza profonda e dialogo interiore è particolarmente intenso quando coinvolge vicende umane significative. La storia umana, vicina e lontana nello spazio come nel tempo, è ricca di queste figure che è doveroso far incontrare alle nuove generazioni. La loro testimonianza, particolarmente pregnante in senso positivo, ma anche, e per contrasto, in senso negativo, è avvicinata dai discenti mediante la comprensione umana, ed essa fa vibrare in loro il desiderio di bene, di giustizia, di libertà, di verità e di bellezza che, tendenzialmente, caratterizza ogni animo umano e così lo conferma, lo rafforza e lo caratterizza come base per un apprendimento antropologicamente radicato.

Si è appena conclusa la lettura di alcuni passi del libro intitolato "La notte» di Elie Wiesel (1980) che racconta gli orrori dei campi di concentramento di Auschwitz. Nella classe c'è silenzio assoluto. Il docente conclude la lezione narrando un'ultima vicenda accaduta nello stesso luogo di morte e di sofferenza. Dal campo si erano verificate delle fughe: per ognuna di loro, i nazisti estrassero a sorte dieci progionieri da condannare a morte nel bunker della fame. Il frate francescano polacco Massimiliano Maria Kolbe (Sicari, 2006) non era tra di loro e tuttavia, da prete, chiese e ottenne di essere sostituito a un padre di famiglia che era stato selezionato per la terribile ritorsione. E nei giorni successivi, il bunker della fame si trasformò, nel campo di concentramento, in un luogo di canti e di speranza religiosa, fino all'ultimo atto.

Un incontro di questa portata può realmente scuotere l'animo. Non si tratta evidentemente di proporre quella di Kolbe come l'unica scelta opportuna in una situazione di una drammaticità nella quale non ci è possibile calarci. Ma la sua scelta di apertura inaudita all'altro non può che toccare in profondità le corde umane di chi viene a conoscerla, confermando quel desiderio di bene che rappresenta la base più solida per una reale educazione ai valori. È una scelta che rappresenta la più forte opposizione all'orrore dei campi di concentramento, luoghi ideati con la precisa intenzione di annientare l'umano in tutte le sue dimensioni. E, è bene sottolinearlo, non si tratta di un incontro che presupponga una fede religiosa: certo, Kolbe era un frate e se il suo sacrificio è stato ispirato dal cristianesimo, il significato di apertura che porta con sé ha un valore umano che può essere inteso da chiunque, come accade, ad esempio, per la lotta non violenta intrapresa da Mahatma Gandhi.

Ricordo una lezione in cui ho presentato l'opposizione al fascismo e la difesa della democrazia pubblicamente attuata dal parlamentare italiano socialista Giacomo Matteotti. Lessi alla classe la dichiarazione di uno dei suoi aguzzini: «Mentre lo pugnalavamo [...] egli ci ripeteva: 'Uccidete me, ma l'idea che è in me non la ucciderete mai!'. [...] Fino alla fine ha gridato: 'La mia idea non muore! I miei bambini si glorieranno del loro padre!'». Dal silenzio, dopo qualche secondo, si è alzata una voce, quella dall'allievo forse più vivace della classe: «Averlo un padre così!». Un'affermazione da cui trapela l'interesse umano suscitato nel giovane da una persona che ha difeso, fino all'ultima soglia, la verità 
e i valori fondamentali. E ciò non implica, naturalmente, che l'allievo si sia votato alla causa del socialismo.

\section{Quella linea, che separa il bene dal male...}

C'è una questione che, a nostro avviso, non dovrebbe essere elusa: la questione del male. Se è vero che nella persona esiste una tensione verso i valori che la tradizione filosofica ha chiamato "trascendentali», è difficile negare che anche l'ingiustizia e l'oppressione, lungo la storia, abbiano esercitato su di essa uno strano fascino. La traduzione di un antico inno cristiano latino ${ }^{8}$ recita: $« D a l$ male che ci affascina il corpo stanco libera». Che lo si voglia chiamare "peccato originale» o "concupiscenza", che lo si voglia associare al corpo o all'anima, si rintraccia facilmente nelle vicende umane questo "lato oscuro" che in numerosi contesti ha determinato l'azione di parti rilevanti delle comunità umane. Si pensi alla facilità che hanno trovato persone come Adolf Hitler o Stalin a circondarsi di adepti propensi a spingere, ancora più là, la violenza delle loro ideologie totalitarie. In questi contesti, la cittadinanza, ovvero "lo stare assieme delle persone», viene asservita con la forza e con la violenza a precise ideologie. Gli allievi sono acuti e intercettano immediatamente i buonismi che censurano il potere esercitato dal male. Intuiscono che qualcosa non quadra e se è così la scuola corre il rischio di essere vissuta come astratta, parziale, sovrapposta alla «vita vera». Del resto, l'integrità di un valore che si giudica come tendenziamente universale è verificata proprio dalla sua capacità di affermarsi di fronte a tutti i fattori della realtà, anche a quelli più drammatici. È proprio qui, del resto, che emergono i limiti dei regimi antidemocratici: da qualche parte, essi sono costretti a nascondere, a insabbiare, a cancellare alcune persone, alcune scelte perché in esse brilla troppo chiaramente la contraddizione e la falsità delle ideologie e dei metodi che propugnano. Lo afferma in termini cristallini Václav Havel:

La copertura della vita nella menzogna è fatta di uno strano materiale: finché chiude ermeticamente tutta la società, sembra essere di pietra, ma appena qualcuno apre uno spiraglio da qualche parte [...], tutto appare improvvisamente sotto un'altra luce e tutta la copertura dà l'impressione di essere di carta e di cominciare a strapparsi in modo inesorabile. (Havel, 2013, p. 58)

Anche in questo caso, è utile avvicinare la tematica con un incontro umano, con chi, queste dinamiche, le ha conosciute sulla propria pelle. Proponiamo qui la figura di Aleksandr Solženicyn del quale si sono recetemente 9 celebrati i cento anni dalla nascita e i dieci anni dalla morte. L'autore russo (1974), nella sua opera più celebre, si pone questa domanda: «Da dove viene questa razza di lupi nel nostro popolo? È di altre radici? È di altro sangue?». La risposta di Solženicyn è perentoria: «No, del nostro» (p. 172). E più avanti precisa: «Si possono dipingere i malvagi nelle storie da bambini, per semplificare il quadro» (p. 184). L'idea del «cattivo» diverso dalle persone comuni, ci dice, può avere un senso per i più piccoli, che necessitano di rassicurazioni, ma essa non è rispondente della realtà. 
Crescendo, occorre che i giovani siano confrontati con un dato di fatto che Solženicyn descrive magistralmente: «La linea che separa il bene dal male attraversa il cuore di ognuno" (p. 179). Tempo fa questa frase era appesa a caratteri cubitali, realizzati dagli allievi, sulla parete dell'aula in cui insegno. Essa porta il discorso sul piano della responsabilità e stimola i ragazzi a intuire che le loro vite sono costellate da scelte, piccole o grandi che siano, cioè occasioni per affermare le proprie convinzioni e i valori fondamentali, perché quello del bene e del male è un problema che tocca sempre chiunque. Ma il discorso deve andare ancora più in là. Solženicyn (1974) si chiede cosa possa spingere qualcuno a optare per la violenza e per l'intolleranza. La risposta lo vede usare alcuni termini da lui redatti integralmente in forma maiuscola: il «NON PENSARE» con la precisazione che questa è «la distruzione di un uomo» (p. 158), il «COMODO» (p. 159); gli istinti del «LUCRO» e il «POTERE» (p. 160). Ci sembra un compendio assai centrato: $\mathrm{i}$ «lupi» sono persone che agiscono senza pensare, che cercano la vita facile e quindi, per non avere problemi, fanno ciò che gli si dice di fare, oppure, che pongono il denaro o il fascino del potere al di sopra dei valori e dei diritti universali. Quanto lavoro si può svolgere in una classe su questi quattro concetti. Un lavoro che rende possibile una maggiore consapevolezza nel momento, che arriverà, in cui i giovani saranno confrontati con queste tentazioni. Un lavoro che si situa nelle profondità dell'umano, proprio là, dove ha le proprie fondamenta la buona cittadinanza.

\section{La valorizzazione della quotidianità}

Sabrina e Laura hanno appena avuto una discussione violenta: hanno alzato la voce, si sono insultate con giudizi di una certa gravità e si ora si stanno squadrando con aria di sfida. Tra di loro c'è una maestra, il volto rosso e l'espressione corrucciata: «Non si litiga! - urla - così disturbate l'ambiente della scuola! Ditemi chi ha iniziato!» Le allieve si indicano a vicenda. "Datevi la mano - riprende l'insegnante - e fate subito la pace!» Le due bambine, si stringono la mano, ma i loro sguardi non s'incrociano.

Il moralismo è così definito dal filosofo e storico della filosofia Nicola Abbagnano (1998): «formalismo morale che ha poca sostanza umana». Esso è un tipico esempio di riduzionismo etico, ovvero di una semplificazione consistente nella rigida formulazione di giudizi morali che non tiene presente la totalità dei fattori implicati in una determinata cirscostanza. Proprio come la distinzione tra «buoni» e «lupi» rigettata da Solženicyn. Qual è il problema tra Sabrina e Laura? Come si sono sentite durante il litigio? Quali esigenze vogliono esprimersi reciprocamente? Come hanno mostrato efficacemente i numerosi contributi di Daniele Novara ed Elena Passerini (2015) ${ }^{10}$ il conflitto non è un'esperienza sbagliata in se stessa, questo vale semmai per la violenza e per gli atti che ledono i valori e i diritti fondamentali, bensì è un'esperienza che può essere generativa, al pari di quanto abbiamo precedentemente osservato a proposito dell'errore, soprattutto se in essa sono mobilitate direttamente le competenze degli allievi. 
Il moralismo semplifica, trascura la persona e quindi, facilmente la umilia producendo effetti opposti a quelli desiderati in un'educazione alla cittadinanza. Piuttosto che imporre rigidamente norme disumanizzate, la maestra della scena descritta pocanzi avrebbe potuto lasciare spazio alla narrazione delle allieve: a turno, ognuna di loro racconta la propria esperienza e l'altra la ascolta, cioè, inizia a lasciarle uno spazio. Per incentivare questo processo, l'insegnante avrebbe anche potuto proporre un'inversione di punto di vista: «Laura, come ti saresti sentita se ti fossi trovata al posto di Sabrina?» E viceversa.

La quotidianità fornisce molteplici spunti per un'educazione alla cittadinanza democratica. La scuola offre a molti bambini la prima occasione per una socializzazione secondaria: per un incontro con un «altro», che non ho scelto io, che si siede accanto a me e che rivela tra lui e me, come tra me e lui, analogie e differenze. A volte, quando nel tempo si è costruita una sufficiente intesa, pongo alle classi alcune domande personali, lasciando la libertà di non rispondere: «Chi è religioso? Nel caso, di quali religioni? Chi non lo è? Chi non lo sa ancora?» Per poi fare notare agli allievi quanto sia preziosa questa convivenza tra analogie e differenze, accoglienza e rispetto; osservando che questo stare assieme non è scontato, ma è stato conquistato grazie al sacrificio e riportando, infine, su di loro la responsabilità della sua conservazione. Quella tra gli allievi non è una semplice "giustapposizione», perché nel gruppo e con il gruppo l'allievo deve lavorare per apprendere, attivando tutte quelle dimensioni della sua persona che abbiamo precedentemente descritto: il muoversi tra il "già» e il "non ancora" lasciando trapelare i propri limiti; il lasciarsi cadere in errore e l'accettare l'aiuto. Vivere soddisfazioni, fatiche e sconfitte. La quotidianità scolastica offre preziose occasioni perché l'autorevolezza dell'adulto dia testimonianza dei valori democratici nella concretezza del lavoro disciplinare: la valorizzazione dell'alterità, la fiducia nell'educabilità e la stima per la persona sono tutte posture che risuonano nell'allievo come profondamente auspicate e così esse vengono apprese attraverso «'interiorizzazione di modelli» (Tuffanelli \& Ianes, 2011, p. 122). In seconda battuta, sono innumerevoli le attività proposte nei libri di pedagogia e di didattica per favorire la creazione del gruppo e lo sviluppo di relazioni positive. Si pensi all'ascolto attivo proposto da Thomas Gordon applicabile alla gestione delle discussioni di gruppo, al consiglio di cooperazione descritto da Danielle Jasmin fino alla Philosophy for Children ideata da Matthew Lippman e a innumerevoli altre proposte sulle quali è opportuno che gli insegnanti si tengano costantemente aggiornati, redigendo eventualmente un proprio diario arricchito dalle esperienze e dalle valutazioni personali. 


\section{Conclusione}

L'educazione può essere definita come un accompagnamento del discente verso un'esperienza più umana della realtà, che richiede la trasmissione di valori fondamentali, connessi a diritti dei quali, tendenzilamente, ogni persona ragionevole desidera godere. La distinzione tradizionale tra «istruzione», intesa come trasmissione d'informazioni e abilità, ed «educazione», intesa come trasmissione di valori, se può avere in certi contesti una propria utilità, nella sostanza risulta una semplificazione: il lavoro, anche strettamente disciplinare, proposto nella scuola è infatti in se stesso educativo come metodo e come contenuti. L'apprendimento è un'esperienza relazionale che pone il discente tra un «già» e un «non ancora», che lo confronta con i propri limiti e con le relazioni sociali, predisponendolo a un'apertura verso il mondo. Esso tocca alcune delle sue dimensioni più strettamente antropologiche: l'intelletto, l'affettività e la libertà. L'apprendimento sviluppa la capacità di rapportarsi con la fatica, con l'ignoto, con le proprie risorse e fragilità, con gli adulti autorevoli (e non) e con i pari. Insegna a sospendere il giudizio svalutante, a rialzarsi dopo le cadute, a riconoscere il valore e la bellezza del lavoro. Tutto ciò rappresenta un bagaglio indispensabile per costruire un'esperienza all'altezza delle possibilità umane: è un lavoro propriamente educativo. Educativi sono anche i contenuti disciplinari che generano un dialogo interiore nel discente e lo rendono capace di un'esperienza del mondo più profonda e più consapevole, sensibile ai valori fondamentali dell'etica $\mathrm{e}$ dell'estetica.

L'educazione alla cittadinanza democratica, intesa come educazione allo stare insieme, richiede un'acquisizione dei valori fondamentali. Ciò avviene principlamente tramite un'educazione prossima alla dimensione antropologica che riveli al discente la corrispondenza tra i valori democratici e le sue più profonde aspirazioni. Sono irrinunciabili, in questo percorso, il confronto con la testimonianza di figure umane significative, la valorizzazione delle relazioni quotidiane e la riflessione sulle motivazioni che possono orientare una persona verso il tradimento dei fondamenti della democrazia.

\section{Note}

1 «L'homme n'est qu'un roseau, le plus faible de la nature, mais c'est un roseau pensant. Il ne faut pas que l'univers entier s'arme pour l'écraser, une vapeur, une goutte d'eau suffit pour le tuer, Mais quand l'univers l'écraserait, l'homme serait encore plus noble que ce qui le tue, puisqu'il sait qu'il meurt et l'avantage que l'univers a sur lui» (Pensées, fr. 231S/347B).

2 Nel Canton Ticino, come altrove, questi diritti dell'insegnante hanno anche una base legislativa: legge della scuola art. 46.

3 Di questa citazione non cogliamo il contrasto tra il mondo fisico e quello razionale che è tipico dell'epoca: abbiamo già precisato che questa separatezza non ci offre una prospettiva adeguata per descrivere e comprendere i processi di apprendimento.

4 L'elenco completo è reperibile sul sito dell'OMS: http://www.who.int

5 L'introduzione di «educazioni specifiche» dovrebbe rispettare un principio di essenzalità nella misura in cui, la loro moltiplicazione, tende a frammentare il lavoro e, paradossal- 
mente, a compromettere la qualità del processo educativo integrale realizzato dalle discipline.

6 Il caso del Canton Ticino è emblematico : dopo un'iniziativa popolare cantonale che ha modificato la legge della scuola nel settembre del 2017, l'educazione civica, alla cittadinanza e alla democrazia, dell'annoscolastico 2018/2019, nella scuola media è stata scorporata dalla storia diventando una materia a sé stante dotata di valutazione specifica. L'insegnamento di questa disciplina ha subito modifiche anche nelle scuole medie superiori.

7 Non ci occuperemo qui di ciò che normalente si definisce con il termine «civica», che ha un significato più tecnico, connesso al funzionamento istituzionale di uno Stato.

8 Traduzione italiana dell'inno "Te lucis ante terminum».

9 Aleksandr Solženicyn è vissuto tra il 1918 e il 2008.

10 Daniele Novara è fondatore e direttore del Centro PiscoPedagogico per l'educazione e la gestione dei conflitti (www.cppp.it) che si occupa esattamente di queste tematiche.

\section{Bibliografia}

Abbagnano, N. (1998). Dizionario di Filosofia, terza edizione aggiornata e ampliata da Giovanni Fornero. Torino, Italia: Utet.

Bellamy, F. (2016). I diseredati: ovvero l'urgenza di trasmettere. Castel Bolognese, Italia: Itaca. Bertola, L. (2015). Parole della vita: per un'educazione all'etica. Trento, Italia: Erickson.

Dewey, J. (1938). Democracy and education in the world of today. In J. A. Boydston (Éd.), John Dewey, The Later Works (pp. 299-303, vol. 13). Carbondale, CO: Southern Illinois University Press.

Dewey, J. (1954). Democrazia e educazione. Firenze, Italia: La Nuova Italia.

Giussani, L. (2005). Il Rischio educativo. Milano, Italia: Rizzoli.

Havel, V. (2013). Il potere dei senza potere. Milano, Italia: Edizioni La Casa di Matriona.

Jungmann, J. A. (1939). Christus als Mittelpunkt religiöser Erziehung. Freiburg, Germania: Herder.

Laffranchini, R. (2015). Il Rischio della libertà: un’esperienza di scuola. Castel Bolognese, Italia: Itaca.

Mariani, A. (2011). Elementi di filosofia dell'educazione. Roma, Italia: Carocci.

Meirieu, P. (2013). I compiti a casa: genitori, figli, insegnanti: a ciascuno il suo ruolo. Milano, Italia: Feltrinelli.

Meirieu, P. (2015). Fare la Scuola, fare scuola: democrazia e pedagogia. Milano, Italia: FrancoAngeli.

Morin, A. (2015). Insegnare a vivere: manifesto per cambiare l'educazione. Milano, Italia: Raffaello Cortina Editore.

Novara, D., \& Passerini, E. (2015). Con gli altri imparo: far funzionare la classe come gruppo di apprendimento. Trento, Italia: Erickson.

Nucci L. P. (2002). Educare il pensiero morale. Trento, Italia: Erickson,

Pascal, B. (16701, 2000). Pensieri. Milano, Italia: Bompiani.

Polito, M. (2015). Educare il cuore: strategie per una comunità che si prende cura delle nuove generazioni. Molfetta, Italia: Edizioni la Meridiana.

Rousseau, J.-J. (1762¹, 2017). Emilio. Roma e Bari, Italia: Editori Laterza.

Sicari, A. (2006). Ritratti di santi, volume 1. Milano, Italia: Jaca Book.

Silva, C. (2015). Lo spazio dell'intercultura: democrazia, diritti umani e laicità. Milano, Italia: FrancoAngeli.

Solženicyn, A. (1974). Arcipelago Gulag (vol. 1). Milano, Italia: Arnoldo Mondadori Editore.

Tuffanelli, L., \& Ianes, D. (2011). La gestione della classe : autorappresentazione, autocontrollo, comunicazione e progettualità. Trento, Italia: Erickson.

Wiesel, E. (19581 , 1980). La Notte. Firenze, Italia: Editrice La Giuntina. 
Parole chiave: Educazione, cittadinanza democratica, antropologia, libertà, coscienza e consapevolezza.

\section{Erziehen, zum Menschsein}

\section{Zusammenfassung}

Dieser Beitrag beginnt mit einer Analyse individueller Lernerfahrungen. Diese Analyse konzentriert sich auf folgende Merkmale: Beziehung, Freiheit, Intellekt und Emotion. Bildung wird als die Hinführung der Lernenden zu einer tieferen menschlichen Erfahrung der Wirklichkeit betrachtet. Diese Definition beinhaltet, dass die konzeptuelle Unterscheidung zwischen Bildung und Erziehung minimiert wird. Bildung ist in gewisser Weise auch erziehend aufgrund ihrer Aufgabe, die eigenen Grenzen kennen zu lernen und sich mit dieser Wirklichkeit auseinander zu setzen. Ebenso sensibilisieren die Bildungsinhalte die Wirklichkeitserfahrungen der Lernenden. Im zweiten Teil des Beitrags wird die Unverzichtbarkeit einer Wertebildung aufgezeigt. Diese wird anhand eines didaktischen Beispiels zur Förderung der Entwicklung eines demokratischen Bewusstseins veranschaulicht.

Schlagworte: Bildung, demokratische Staatsbürgerschaft, Anthropologie, Freiheit, , Gewissen, Bewusstsein.

\section{Eduquer à être hommes}

\section{Résumé}

Cette contribution commence par une analyse de l'expérience de l'apprentissage focalisée sur les aspects suivants : la relation, la liberté, l'intellect et la sphère affective. L'éducation est ensuite définie comme l'accompagnement de l'apprenant.e vers une expérience plus profondément humaine de la réalité. Cette définition oblige à considérer comme réductrice la distinction conceptuelle entre instruction et éducation. L'instruction est en effet elle-même éducative par sa méthode, qui porte l'élève à se confronter à ses propres limites et à s'ouvrir à la réalité. De même les contenus de l'instruction enrichissent l'expérience de l'élève en la rendant plus consciente de la réalité. Dans la deuxième partie de l'article, nous montrerons que, dans ce parcours, l'éducation aux valeurs devient indispensable. Une proposition didactique d'éducation à la citoyenneté démocratique fournira un exemple de mise en pratique.

Mots clés: Éducation, citoyenneté démocratique, anthropologie, liberté, conscience 


\section{Educate to be human}

\section{Summary}

The article begins with an analysis of the learning experience focused on the following essential elements: relationships, freedom, intellect and emotions. Education is then defined as accompanying the learner towards a more deeply human experience of reality. This definition requires judging the conceptual distinction between education and instruction as reductive. Instruction is, in fact, itself educating for the method, which teaches you to confront your limits and favors being open-minded.

As for the content, education makes the experience of reality have a greater awareness. In this process, educating to values is essential. This subject is studied in the second part of the article with a didactic proposal of education for democratic citizenship.

Keywords: Democratic citizenship, anthropology, freedom, conscience and awareness

\section{Autore}

Raffaele Beretta Piccoli, MA in Filosofia delle religioni. Insegnante alla Scuola Media di Caslano (TI) e docente di filosofia dell'educazione alla Scuola Universitaria Professionale della Svizzera Italiana.

Scuola Universitaria Professionale della Svizzera Italiana, Piazza S. Francesco 19, CH-6600 Locarno

E-Mail: raffaele.berettapiccoli@supsi.ch 